\title{
Posición de las terceras molares mandibulares según la clasificación de Pell y Gregory en radiografías panorámicas en un Centro Radiológico De Huancayo - Perú 2018.
}

Frequent position of third mandibular molars, according to Pell and Gregory classification in panoramic $\mathrm{x}$ rays at the -ZERO, Huancayo 2018

Flor de María Apumayta De La Cruz ${ }^{1, a}$, Ruth Edibirges Lara Rivera ${ }^{1, a}$, Jhon Rondinel Berna 1,b

\section{RESUMEN}

Objetivo: Determinar cuál es la prevalencia de la posición de las terceras molares mandibulares, según la clasificación de Pell y Gregory en radiografías panorámicas en un Centro Radiológico, Huancayo 2018. Material y métodos: La metodología empleada por el periodo de secuencia de estudios, es transversal retrospectivo y según su nivel descriptiva. El muestreo fue no probabilístico haciendo el uso del método por conveniencia, conformada por 396 radiografías panorámicas en un rango de edad de 18 a 40 años. Resultados: De un total 792 terceras molares mandibulares, excluyendo las piezas ausentes, existe mayor prevalencia de la clase II posición B con un 37,5\% $(\mathrm{n}=297)$, seguido por la clase II posición A con un $22,5 \%(\mathrm{n}=178)$ y clase I posición A con un $18,8 \%(\mathrm{n}=149)$ del total. Conclusiones: Existe mayor prevalencia de la clase II posición B, en ambos sexos, grupos etarios y lados (D-I) en las radiografías panorámicas evaluadas.

PALABRAS CLAVE: Terceras molares mandibulares, radiografías panorámicas, clasificación de Pell y Gregory.

\section{SUMMARY}

Objective: To determine the most frequent position of third mandibular molars, according to Pell and Gregory classification in panoramic $\mathrm{x}$ - rays at the -ZERO, Huancayo 2018. Material and methods: Regarding the methodology used by the sequence of studies period, it is retrospective crosssectional and according to its level it was descriptive. The sample was not probabilistic, for convenience, consisting of 396 panoramic radiographs in an age range of 18 to 40 years. Results: A total 792 third mandibular molars, excluding missing parts, there is a higher prevalence of class II position B with 37.5 percent $(\mathrm{n}=297)$, followed by class II position A with 22.5 percent $(\mathrm{n}$ $=178)$ and class I position A with 18.8 percent $(n=149)$ of the total. Conclusions: There is a higher prevalence of class II position B, in both sexes and in most age groups in the panoramic x-rays evaluated.

KEYWORDS: Third mandibular molars, panoramic radiographs, Pell and Gregory classification.

Facultad de Ciencias de la Salud Escuela Profesional de Estomatología, Universidad Privada De Huancayo Franklin Roosevelt. Huancayo, Perú.

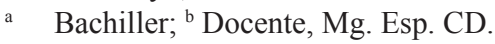




\section{INTRODUCCIÓN}

Entre las cirugías que con mayor frecuencia realiza el odontólogo general en su práctica diaria, se encuentran las exodoncias simples y complejas de las terceras molares mandibulares indicadas por causas como, falta de espacio, infecciones, pericoronitis, mala posición, autotrasplante, periodontitis, caries y, en algunos casos, por estar relacionadas con quistes o tumores, entre otras patologías.

La radiografía panorámica se considera de gran utilidad en el diagnóstico oral y máxilo facial, permitiendo observar todos los dientes, el maxilar, la mandíbula y otros componentes anatómicas adyacentes, antes que se realicé un procedimiento quirúrgico y debería ser de norma general pedirla para el diagnóstico.

La indicación para la remoción quirúrgica de un diente impactado o incluido, debe ser considerada después de evaluar los factores relacionados a estos dientes. Por eso, para realizar la intervención quirúrgica es necesario elaborar un correcto plan de tratamiento, que varía en función de la posición del diente no erupcionado. Para facilitar el planeamiento quirúrgico, surgieron algunos sistemas de clasificación de las terceras molares mandibulares impactadas o incluidas que permiten la anticipación de posibles trastornos y posibilitan la previsión de algunas modificaciones durante el acto operatorio. Estas clasificaciones fueron hechas a partir de análisis radiográficos y en la gran mayoría de veces, se utilizan radiografías panorámicas, donde es posible visualizar correctamente el eje longitudinal del segundo molar, rama de la mandíbula y el nivel óseo que sirven como parámetros (1).

La falta de investigaciones sobre el tema en esta región del país, amerita una investigación sobre la posición de las terceras molares mandibulares impactadas e incluidas, respecto a la clasificación de Pell \& Gregory, según su profundidad en relación a la segunda molar (Posición A, B ó C) y su relación con la rama

ascendente mandibular y la segunda molar (Clase I, II ó III) (2).

Relación del Cordal con Respecto a la rama Ascendente de la Mandíbula y el Segundo Molar (Figura 1)

Clase I. Existe suficiente espacio entre la rama ascendente de la mandíbula y la parte distal del segundo molar para albergar todo el diámetro mesiodistal de la corona del tercer molar.

Clase II. El espacio entre la rama ascendente de la mandíbula y la parte distal del segundo molar es menor que el diámetro mesiodistal de la corona del tercer molar.

Clase III. Todo o casi todo el tercer molar está dentro de la rama ascendente de la mandíbula.

\section{Profundidad Relativa del Tercer Molar en el Hueso (Figura 2)}

Posición A. El punto más alto del diente incluido está al nivel, o por arriba, de la superficie oclusal del segundo molar.

Posición B. El punto más alto del diente se encuentra por debajo de la línea oclusal, pero por arriba de la línea cervical del segundo molar.

Posición C. El punto más alto del diente está al nivel, o debajo, de la línea cervical de la segunda molar.
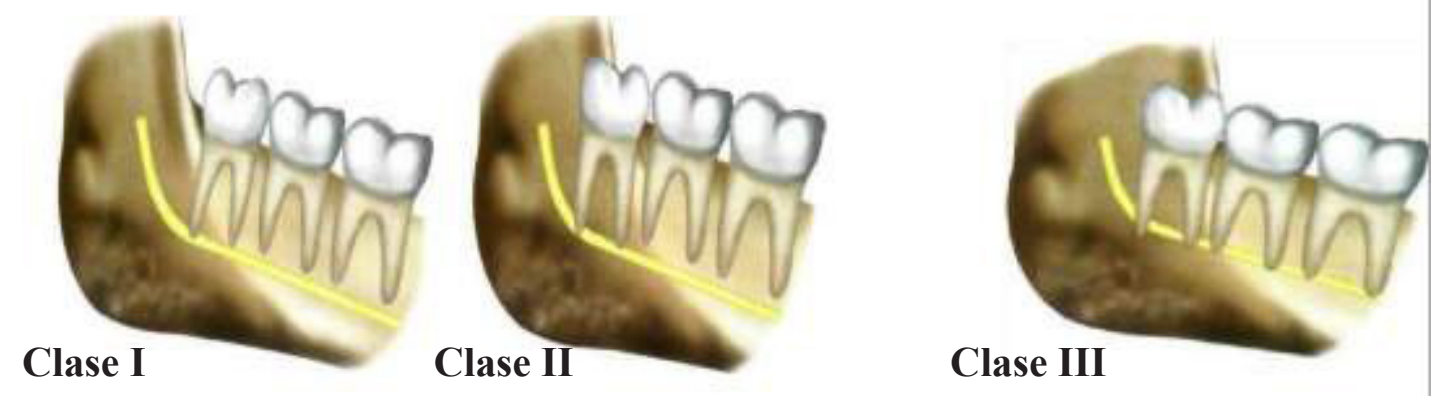

Figura 1. Relación entre el tercer molar y la rama ascendente de la mandíbula (clasificación de Pell y Gregory). 


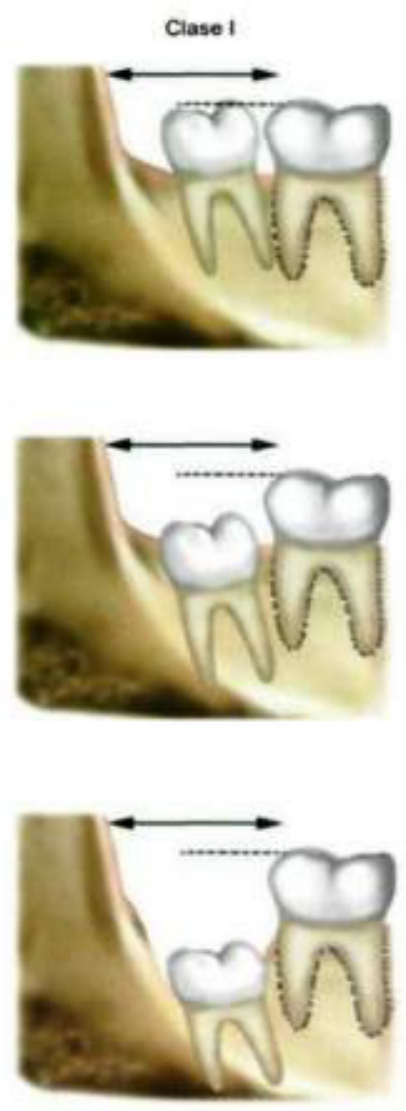

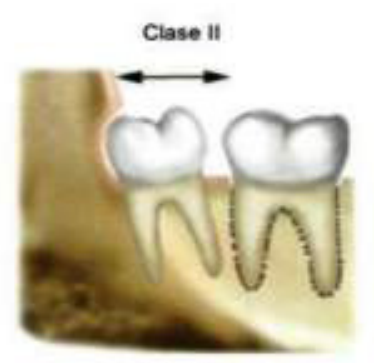

Posición A

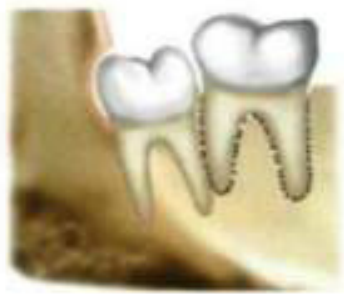

Posicion B

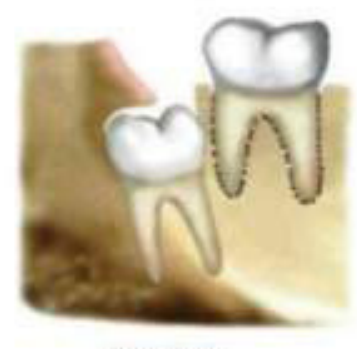

Posicion C
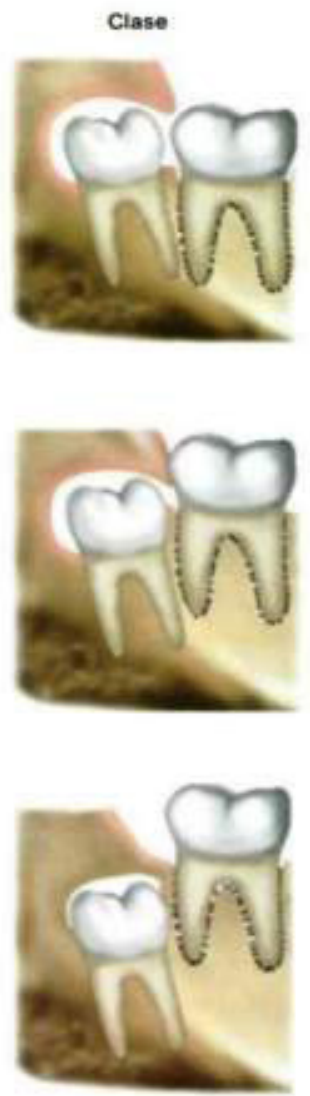

Figura 2. Clasificación de Terceros molares inferiores.

El objetivo del presente estudio fue determinar cuál es la posición más frecuente de las terceras molares mandibulares según la clasificación de Pell y Gregory en radiografías panorámicas, en el Centro Especializado de Radiología Odontológica - CERO, Huancayo 2018.

\section{MATERIAL Y METODOS}

Se empleó el método de la observación científica, en las radiografías panorámicas.

Con un tipo y nivel de investigación según de la planificación de la toma de datos es retrospectivo, transversal y de nivel descriptivo.

Se realizó un diseño epidemiológico no experimental; donde se evaluaron radiografías panorámicas de la base de datos del Centro Especializado de Radiología Odontológica CERO, Huancayo 2018.
La población de estudio estuvo conformada por todas las radiografías panorámicas tomadas a los pacientes que acudieron al Centro Especializado de Radiología Odontológica - CERO, Huancayo, en el año 2018.

El muestreo fue no probabilístico haciendo uso del método de conveniencia e incluyó todas aquellas radiografías panorámicas tomadas a pacientes en el rango de 18 a 40 años, de ambos sexos, que cumplieron con los criterios de inclusión y exclusión, obteniendo un total de 396.

\section{Criterios de inclusión}

- Radiografías panorámicas de pacientes con edades entre 18 a 40 años de edad de ambos sexos.

- Radiografías panorámicas de pacientes que presenten terceras molares inferiores, derecha y/o izquierda con raíz completa.

- Radiografías panorámicas de pacientes que presenten segunda molar inferior adyacente. 


\section{Criterios de exclusión}

- Radiografías panorámicas de pacientes con Síndromes y/o enfermedades congénitas.

- Radiografías panorámicas de pacientes con tratamiento de ortodoncia.

- Radiografías panorámicas de pacientes que presenten patologías como enfermedad periodontal, quistes y tumores.

- Radiografías panorámicas de pacientes que presenten ausencia de primer molar inferior con la subsecuente mesialización de la segunda molar inferior.

Técnicas e instrumentos de recolección de datos.

La observación científica. El instrumento empleado fue la ficha de recolección de datos, con los parámetros correspondientes según la clasificación de Pell y Gregory.

Se seleccionaron todas las radiografías panorámicas que cumplieron con los criterios de inclusión en total 396.

Con la radiografía panorámica se registró edad, género y el análisis radiográfico de las terceras molares inferiores; según la clasificación de Pell y Gregory.

Con los datos obtenidos a través de las fichas de observación se registró en el Programa Microsoft Office Excel 2017. Para luego ser analizados por estadística descriptiva en el programa SPSS V.20. Las variables cualitativas utilizaron el estadístico de prueba frecuencias absolutas y relativas, en tanto que para las variables cuantitativas se realizaron estimaciones puntuales y por intervalos de confianza de $95 \%$ y un margen de error menos al 5\%.

\section{RESULTADOS}

A continuación, se analizaron los resultados siguiendo los objetivos planteados, se utilizó fichas individuales impresas y llenadas a mano. Los datos para nuestra investigación fueron obtenidos de las radiografías panorámicas de la base de datos del Centro Especializado de Radiología odontológica CERO. Luego, se registró en el programa Microsoft Office Excel 2017, para luego ser analizados por estadística descriptiva en el programa SPSS para Windows en su versión V.20 (SPSS INC.Chicago IL, USA).
En la tabla y se puede observar que el 56,31\% de la muestra de radiografías panorámicas es del sexo femenino y el $43,69 \%$ son del sexo masculino.

En la tabla 2 se puede observar que de las 396 radiografías panorámicas el $53,5 \%(\mathrm{n}=212)$ de la muestra corresponde al grupo etario de 18 a 24 años de edad, el $31,3 \%(n=124)$ al grupo etario de 25 a 30 años y el $9.3 \%(n=37)$ al grupo etario de 31 a 35 años y por ultimo con un $5.8 \%(\mathrm{n}=23)$ al de 36 a 40 años.

En la tabla 3 se observa de un total de 792 terceras molares mandibulares, excluyendo las piezas ausentes, con un 37,5\% $(\mathrm{n}=297)$ tiene mayor prevalencia la clase II posición B; seguido por la clase II posición A con

Tabla 1. Frecuncia porcentual de las radiografías panorámicas según sexo

\begin{tabular}{ccc}
\hline Sexo & n & Porcentaje \\
\hline Masculino & 173 & 43.7 \\
Femenino & 223 & 56.3 \\
Total & 396 & 100 \\
\hline
\end{tabular}

Tabla 2. Frecuencia porcentual según grupo etareo.

\begin{tabular}{lcc}
\hline & n & Porcentaje \\
\hline 18 a 24 años & 212 & 53.5 \\
25 a 30 años & 124 & 31.3 \\
31 a 35 año & 37 & 9.3 \\
36 a 40 años & 23 & 5.8 \\
Total & 396 & 100 \\
\hline
\end{tabular}

Tabla 3. Frecuencia Porcentual de la variable posición de las terceras molares mandibulares.

\begin{tabular}{lcc}
\hline & n & Porcentaje \\
\hline Ausencia de pieza dentaria & 72 & 9.1 \\
Clase I, Posición A & 149 & 18.8 \\
Clase I, Posición B & 40 & 5.1 \\
Clase II, Posición A & 178 & 22.5 \\
Clase II, Posición B & 297 & 37.5 \\
Clase II, Posición C & 5 & 0.6 \\
Clase III, Posición B & 37 & 4.7 \\
Clase III, Posición C & 14 & 1.8 \\
Total & 792 & 100 \\
\hline
\end{tabular}


un $22,5 \%(\mathrm{n}=178)$ y clase I posición A con un $18,8 \%$ $(\mathrm{n}=149)$ de la muestra, así también podemos observar que el 9,1\% $(n=72)$ de las terceras molares estuvieron ausentes.

En la tabla 4 se puede observar mayor prevalencia de clase II posición B en ambos sexos, de 792 terceras molares mandibulares, excluyendo las piezas ausentes $(\mathrm{n}=72)$. En el sexo femenino con un $23.48 \%(\mathrm{n}=186)$ mientras que en el sexo masculino con un $14.02 \%$ $(\mathrm{n}=111)$ que representan a la misma clasificación.

En la tabla 5 se encontró que del total de 792 molares mandibulares con un $37.5 \%(\mathrm{n}=297)$, excluyendo las piezas ausentes $(\mathrm{n}=72)$, la prevalencia de la clase II posición $\mathrm{B}$ en todos los grupos a excepción del grupo etario de 31 a 35 año, donde la clase I posición A tuvo mayor predominio.
En la tabla 6 se puede observar mayor prevalencia de la clase II posición B con un $35,6 \%(n=141)$ en el lado derecho de ambos sexos, seguido por la clase I posición A con un $21,2 \%$ y clase II posición A con un $20,71 \%$ de la muestra, así también podemos observar que el $10,6 \%$ de las terceras molares del lado derecho estuvieron ausentes.

En la tabla 7 se puede observar mayor prevalencia de la clase II posición $\mathrm{B}$, con un 39,39\% $(\mathrm{n}=156)$, seguido por la clase II posición A con un $24,24 \%(n=96)$ y clase I posición A con un $16,41 \%(\mathrm{n}=65)$ de la muestra. Asimismo, podemos observar que el $7,58 \%(\mathrm{n}=30)$ de las terceras molares mandibulares del lado izquierdo estuvieron ausentes.

Tabla 4.Frecuencia procentual de la posición de las terceras molares mandibulares en cuanto al sexo.

\begin{tabular}{lcccccc}
\hline & \multicolumn{2}{c}{ Masculino } & \multicolumn{2}{c}{ Femenino } & & \\
\cline { 2 - 5 } & D-I & $\mathbf{\%}$ & D-I & $\mathbf{\%}$ & Total & \% \\
\hline Ausencia de pieza dentaria & 35 & 4.42 & 37 & 4.67 & 72 & 9.1 \\
Clase I, Posición A & 70 & 8.84 & 79 & 9.97 & 149 & 18.8 \\
Clase I, Posición B & 14 & 1.77 & 26 & 3.28 & 40 & 5.1 \\
Clase II, Posición A & 85 & 10.73 & 93 & 11.74 & 178 & 22.5 \\
Clase II, Posición B & 111 & 14.02 & 186 & 23.48 & 297 & 37.5 \\
Clase II, Posición C & 3 & 0.38 & 2 & 0.25 & 5 & 0.6 \\
Clase III, Posición B & 20 & 2.53 & 17 & 2.15 & 37 & 4.7 \\
Clase III, Posición C & 8 & 1.01 & 6 & 0.76 & 14 & 1.8 \\
Total & 346 & 43.69 & 446 & 56.31 & 792 & 100
\end{tabular}

Tabla 5. Frecuencia porcentual de la posición de las terceras molares mandibulares en los grupos etarios.

\begin{tabular}{|c|c|c|c|c|c|c|c|c|c|c|}
\hline & \multicolumn{2}{|c|}{18 a 24 años } & \multicolumn{2}{|c|}{25 a 30 años } & \multicolumn{2}{|c|}{31 a 35 años } & \multicolumn{2}{|c|}{36 a 40 años } & \multicolumn{2}{|c|}{ Total } \\
\hline & $\mathrm{n}$ & $\%$ & $\mathbf{n}$ & $\%$ & n & $\%$ & $\mathbf{n}$ & $\%$ & $\mathbf{n}$ & $\%$ \\
\hline $\begin{array}{l}\text { Ausencia de pieza } \\
\text { dentaria }\end{array}$ & 33 & 4.17 & 19 & 2.4 & 13 & 1.64 & 7 & 0.88 & 72 & 9.1 \\
\hline Clase I, Posición A & 62 & 7.83 & 48 & 6.06 & 26 & 3.28 & 13 & 1.64 & 149 & 18.8 \\
\hline Clase I, Posición B & 25 & 3.16 & 12 & 1.52 & 2 & 0.25 & 1 & 0.13 & 40 & 5.1 \\
\hline Clase II, Posición A & 103 & 13.01 & 59 & 7.45 & 12 & 1.52 & 4 & 0.51 & 178 & 22.5 \\
\hline Clase II, Posición B & 179 & 22.6 & 91 & 11.49 & 13 & 1.64 & 14 & 1.77 & 297 & 37.5 \\
\hline Clase II, Posición C & 2 & 0.25 & 1 & 0.13 & 0 & 0 & 2 & 0.25 & 5 & 0.6 \\
\hline Clase III, Posición B & 17 & 2.15 & 14 & 1.77 & 4 & 0.51 & 2 & 0.25 & 37 & 4.7 \\
\hline Clase III, Posición C & 3 & 0.38 & 4 & 0.51 & 4 & 0.51 & 3 & 0.38 & 14 & 1.8 \\
\hline Total & 424 & 53.54 & 248 & 31.31 & 74 & 9.34 & 46 & 5.81 & 792 & 100 \\
\hline
\end{tabular}


Tabla 6.Frecuencia porcentual de la posición de las terceras molares mandibulares lado derecho.

\begin{tabular}{lcc}
\hline & n & \% \\
\hline Ausencia de pieza dentaria & 42 & 10.6 \\
Clase I, Posición A & 84 & 21.2 \\
Clase I, Posición B & 24 & 6.1 \\
Clase II, Posición A & 82 & 20.7 \\
Clase II, Posición B & 141 & 35.6 \\
Clase II, Posición C & 1 & 3 \\
Clase III, Posición B & 15 & 3.8 \\
Clase III, Posición C & 7 & 1.8 \\
Total & 396 & 100 \\
\hline
\end{tabular}

Tabla 7. Frecuencia porcentual de la posición de las terceras molares mandibulares lado izquierdo

\begin{tabular}{lcc}
\hline & n & \% \\
\hline Ausencia de pieza dentaria & 30 & 7.6 \\
Clase I, Posición A & 65 & 16.4 \\
Clase I, Posición B & 16 & 4 \\
Clase II, Posición A & 96 & 24.2 \\
Clase II, Posición B & 156 & 39.4 \\
Clase II, Posición C & 4 & 1 \\
Clase III, Posición B & 22 & 5.6 \\
Clase III, Posición C & 7 & 108 \\
Total & 396 & 100 \\
\hline
\end{tabular}

\section{DISCUSIÓN}

Una vez presentado los resultados de las pruebas estadísticas realizadas y de su interpretación, considerando los objetivos planteados en la investigación y la información hallada en la literatura. Es necesario resaltar que la Clasificación de Pell \& Gregory, establece la relación de las terceras molares respecto al espacio disponible para su erupción entre el segundo molar y la rama ascendente de la mandíbula, además de su profundidad relativa en el hueso y su relación con el segundo molar (3). Por lo tanto, determinar la posición de las terceras molares mandibulares en nuestra población permitirá contar con una base de datos que servirá como referencia para un mejor planeamiento quirúrgico, evitando futuras complicaciones logrando que el paciente tenga una atención odontológica de excelente calidad con menor tiempo y sin traumas posquirúrgicos.
Como se puede apreciar en la tabla 3, de las 396 radiografías panorámicas, se encontró un total de 792 terceras molares excluyendo piezas dentales ausentes; obteniendo que la posición más frecuente de las terceras molares mandibulares, fue la clase II posición $\mathrm{B}$, representado con el 37,5\%. $(\mathrm{n}=297)$. Estos resultados tienen similitud con el estudio de Bustamante N. (2017) (4), en su tesis cuyo objetivo fue determinar la frecuencia de la posición de terceros molares mandibulares según la clasificación de Winter y Pell \& Gregory en radiografías panorámicas digitales de pacientes de 18 a 40 años, donde la posición más frecuente fue la clase II, posición B con un $36.32 \%$, tal como figura en la tabla 7. De igual forma Chávez N. (2012)(5), cuyo objetivo fue determinar la ubicación de la posición de las terceras molares mandibulares según la Clasificación de Winter y según la Clasificación de Pell y Gregory en pacientes de 18 a 50 años, donde la clase II posición $\mathrm{B}$ fue la más representativa con un $40.01 \%$. También Se encontró similitud con lo señalado por Sthorayca F. (2017) (6). En su investigación que tuvo como objetivo determinar la frecuencia de los terceros molares inferiores retenidos en radiografías panorámicas digitales empleando la clasificación de Pell y Gregory y la de Winter, donde encontró como resultado en ambos lados derecha e izquierda, género masculino y femenino mayor frecuencia de la clase II con un $71.3 \%$ y el tipo B con un $51 \%$. A si mismo los resultados hallados en nuestro trabajo de investigación difiere con lo hallado en Huánuco por Pozo K. (2016) (7). En su tesis Posición del Tercer Molar en Relación a la Ubicación del canal mandibular, en pacientes adultos jóvenes de 19 a 25 á. de ambos sexos según la clasificación de Pell y Gregory; de una muestra de 30 radiografías panorámicas de los centros radiológicos CERO y CEDIDENT. Obtuvo como resultado la Prevalencia de la Clase III Posición C con un 36.7\%; ambos sexos y edad.

Con respecto a la prevalencia de la posición de las terceras molares mandibulares según la clasificación de Pell y Gregory en radiografías panorámicas, en cuanto al sexo; tal como figura en la tabla 4 se obtuvo como resultado que la posición fue la clase II posición $\mathrm{B}$, tanto en el sexo femenino como masculino. En el sexo femenino, de un total de 186 terceras molares mandibulares, con el $23.48 \%$ representa a la Clase II y posición $\mathrm{B}$, mientras que, en el sexo masculino, de un total de 111 terceras molares mandibulares el 14.02\% representan a la misma clasificación. Estos resultados tienen semejanza con la investigación de Chávez $\mathrm{N}$. (2012)(5), cuyo objetivo de su tesis fue determinar la ubicación de terceras molares inferiores según la 
clasificación de Winter y la clasificación de Pell y Gregory en radiografías panorámicas de pacientes entre 18 a 50 años de la clínica odontológica de la universidad privada Norbert Wiener, en su trabajo predominó la clase II, posición B con un $40.1 \%$, de ello, el 18,8\% corresponde al sexo femenino y el $21,3 \%$ al sexo masculino, siendo este último grupo donde se aprecia mayor prevalencia, a diferencia de nuestro trabajo, donde el mayor predominio estuvo en el sexo femenino, como ya se hizo referencia.

Con respecto a la prevalencia de la posición de las terceras molares mandibulares según la clasificación de Pell y Gregory en radiografías panorámicas, en los grupos etarios, en la tabla se encontró que la clase II posición B fue la más prevalente en todos los grupos con un $37.5 \%(\mathrm{n}=297)$ a excepción del grupo etario de 31 a 35 año, donde la clase I posición A tuvo mayor predominio; estos resultados tienen semejanza con la investigación de Chávez N. (2012)(5), donde la Clase II P B obtuvo el predominio con un $27.2 \%$ en el grupo etario de 18 a 24 años. Estos resultados encontrados son similares en cuanto a la clase, pero difieren en relación a la posición los hallazgos de Ortiz F, Tobar N. (2017) (8), donde su objetivo fue determinar la frecuencia de posición de terceros molares mandibulares en radiografías panorámicas en pacientes entre 18 y 25 años de ambos géneros, donde prevaleció la clase II posición A con un 39\% en casi todas las edades. A si mismo nuestros resultados difiere en cuanto a posición pero tienen similitud en relación a la clase, con el trabajo de investigación de Días E. y et al. (2009)(1). Donde tuvieron como objetivo realizar un levantamiento radiográfico, utilizando radiografías panorámicas para evaluar la posición de terceros molares inferiores. Los resultados encontrados mostraron que pacientes, con edad entre 20-25 años, fueron los más afectados por la retención del tercer molar. Según la clasificación utilizada, existía un predominio de la Clase II posición A.

Con respecto a la prevalencia de la posición de las terceras molares mandibulares según la clasificación de Pell y Gregory en radiografías panorámicas, lado derecho e izquierdo, en la tabla 6 de nuestro trabajo de investigación se puede observar que la clase II posición $B$ en el lado derecho con un 35,6\% ( $\mathrm{n}=141)$; con respecto al lado izquierdo en la tabla 7 se puede observar mayor prevalencia de la clase II posición B con un $39,4 \%(n=156)$, estos resultados son similares a lo hallado por Meza W (2017)(9), en su tesis, donde el objetivo fue determinar la prevalencia en pacientes que presenten terceros molares impactados con diferentes inclusiones en el maxilar superior e inferior. De igual manera describir la posición de las terceras molares según la clasificación de Winter y Pell y Gregory; los resultados respecto a la clasificación de Pell y Gregory en el maxilar inferior derecho fueron de predominio de la clase II con un $51,2 \%$ y la posición B con un $40.0 \%$; con respecto al maxilar inferior izquierdo obtuvo como resultado la clase II con un $46,2 \%$ y la posición $\mathrm{B}$ con un $52,5 \%$. Estos resultados son similares con el estudio de Serrano J. (2018) (10), que tuvo como objetivo determinar la relación de posición de terceras molares inferiores y su patología asociada en pacientes de 18 a 30 años que acuden a la clínica docente estomatológica de la universidad Alas Peruanas en el año 2018, obteniendo como resultado una mayor prevalencia, en el lado izquierdo, de la clase II con $51,7 \%$ y el tipo con mayor prevalencia fue la B con 33,9\%; y en el lado derecho la clase II con un $50,8 \%$ y la posición A con $44,9 \%$ siendo este último resultado con respecto a la posición diferente a nuestro trabajo investigación. También se halló que nuestros resultados difieren con respecto al predominio del lado izquierdo con Tirado J. (2015)(11), quien en su tesis consideró como objetivo determinar la posición más frecuente de las terceras molares mandibulares según el factor género y la clasificación de Pell y Gregory en pacientes de 18 a 40 años, dando como resultado la clase II, posición B en el lado derecho con $39,2 \%$ y con un $37,7 \%$ en el lado izquierdo.

\section{CONCLUSIONES}

Se halló que la clase II, posición B tiene mayor prevalencia con un $37,5 \%(\mathrm{n}=297)$, seguido por la clase II posición A con un $22,5 \%(\mathrm{n}=178)$ y clase I posición A con un 18,8\% ( $\mathrm{n}=149)$, según la clasificación de Pell \& Gregory en radiografías panorámicas.

Se encontró que la posición de las terceras molares mandibulares, tuvo mayor prevalencia la clase II posición $\mathrm{B}$ en ambos sexos; en el sexo femenino con un $23.48 \%$ y en el sexo masculino con un $14.02 \%$, de acuerdo a la clasificación de Pell y Gregory.

Se ha identificado que la posición de las terceras molares mandibulares, según la clasificación de Pell y Gregory, tuvo mayor prevalencia la clase II posición B en la mayoría de los grupos etarios, siendo mayor en el grupo etario de 18 a 24 años con un 37,5\% radiografías panorámicas.

Se encontró la prevalencia según la clasificación de Pell y Gregory,la clase II 
posición $\mathrm{B}$, en el lado derecho con un $35.06 \% \mathrm{y}$ en el lado izquierdo con un $39.40 \%$, en las radiografías panorámicas.

Agradecimientos: Al Mg. Rodrigo E. Salazar Lazo, por facilitarnos la base de datos del Centro Especializado de Radiología Odontológica - CERO, Huancayo 2018.

\section{Correspondencia}

Jhon Rondinel Berna

Av. Giráldez 542, Huancayo 12001.

Junin, Perú

\section{REFERENCIAS BIBLIOGRÁFICAS}

1. Días E.. Prevalencia de la posición de terceros molares inferiores retenidos con relación a la clasificación de Pell \& Gregory. ROM. 2009; 13: pp 229-233.

2. Ibarra F. Frecuencia de la localización de las terceras Molares Inferiores en Radiografías Panorámicas de Pacientes Atendidos en el centro de Atención Ambulatorio Central Guayas. [Tesis de Licenciatura]. [Guayaquil - Ecuador]: Universidad Cayetano Heredia; 2014.

3. Gay C, Berini L. Tratado de Cirugía Bucal. Tomo I. Barcelona, España: Océano/Ergon; 2004. 355-407 p.

4. Bustamante N. "Frecuencia de la posición de los terceros molares mandibulares según la clasificación de Winter y Pell \& Gregory en radiografías panorámicas digitales de pacientes de 18 a 40 años que acudieron al centro radiológico de la Universidad Católica de Cuenca durante el periodo Septiembre 2016 - Octubre 2017." [España]: Universidad Católica de Cuenca; 2017.

5. Chávez N. Ubicación de terceras molares inferiores según la clasificación de Winter y la clasificación de Pell y Gregory en radiografías panorámicas de pacientes entre 18 a 50 años de la clínica odontológica de la universidad privada Norbert Wiener, en el periodo 2012-2014, Lima - Perú.
6. Sthorayca F. Frecuencia de Terceros Molares Inferiores Retenidos según la Clasificación de PellGregory y la Clasificación de Winter; de Acuerdo a la Hemiarcada y Género en Radiografías Panorámicas Digitales de Pacientes Atendidos en un Centro de Diagnóstico por Imágenes de la Ciudad de Tacna, Rev.ET Vita 2017; Vol 12-N 2.

7. Pozo K. Posición del tercer molar en relación a la ubicación del canal mandibular en pacientes adultos jóvenes de la ciudad de Huánuco.

8. Ortiz F. y Tobar N. Frecuencia de posición de terceros molares inferiores en radiografías panorámicas de la Universidad Finis Terrae en el año 2017 [Internet] [Thesis]. [Chile]: Universidad Finis Terrae (Chile) Facultad de Odontología; 2017 [citado 18 de mayo de 2019]. Disponible en: http://repositorio.uft.cl:80/xmlui/ handle/20.500.12254/790

9. Meza W. Prevalencia y clasificación de los terceros molares incluidos en pacientes que acudan al quirófano de la facultad de odontología de la UDLA durante el periodo septiembre-octubre 2017 [Internet]. [Quito,Ecuador]: UDLA; 2017 [citado 3 de junio de 2019]. Disponible en: http://dspace.udla.edu.ec/ handle $/ 33000 / 8540$

10. Serrano J. (2018) En su trabajo de Investigación Frecuencia en la posición de terceras molares inferiores y su patología asociada en pacientes de 18 a 30 años que acuden a la clínica docente estomatológica de la universidad alas peruanas en el año 2018.

11. Tirado J. Posición más frecuente de terceras molares mandibulares según la clasificación de Pell y Gregory con relación al factor género en el Hospital Central Fap [Internet]. [Lima - Perú]: Universidad Nacional Mayor de San Marcos; 2015 [citado 18 de mayo de 2019]. Disponible en: http://cybertesis.unmsm.edu. pe/handle/cybertesis/4273

Recibido: 18/01/2020

Aceptado: 16/07/2020 